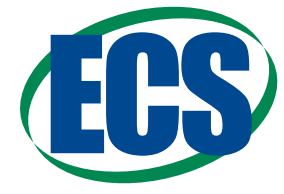

Jes Focus Issue on Mathematical Modeling of Electrochemical Systems at Multiple Scales in Honor of John Newman

\title{
Impedance Spectroscopy Study of the PEM Fuel Cell Cathode with Nonuniform Nafion Loading
}

\author{
Tatyana Reshetenko ${ }^{\mathrm{a}, *, \mathrm{z}}$ and Andrei Kulikovsky ${ }^{\mathrm{b}, \mathrm{c}, *, \mathrm{z}}$ \\ ${ }^{a}$ Hawaii Natural Energy Institute, University of Hawaii, Honolulu, Hawaii 96822, USA \\ ${ }^{b}$ Institute of Energy and Climate Research, Electrochemical Process Engineering, Forschungszentrum Juelich GmbH, \\ D-52425 Jülich, Germany \\ ${ }^{c}$ Research Computing Center, Lomonosov Moscow State University, 119991 Moscow, Russia
}

We report modeling and experimental study of impedance of the PEM fuel cell cathode with nonuniform ionomer loading. A physics-based model for the high-frequency impedance is developed and analytical solution for impedance is derived. Assuming that the CCL proton conductivity $\sigma_{p}$ exponentially decays from the membrane surface, we fit the model to experimental spectra of the cell measured at the open circuit conditions. Fitting gives the characteristic scale of the $\sigma_{p}$ decay, the average CCL proton conductivity and the double layer capacitance.

(C) The Author(s) 2017. Published by ECS. This is an open access article distributed under the terms of the Creative Commons Attribution 4.0 License (CC BY, http://creativecommons.org/licenses/by/4.0/), which permits unrestricted reuse of the work in any medium, provided the original work is properly cited. [DOI: 10.1149/2.0041711jes] All rights reserved.

(cc) BY

Manuscript received January 10, 2017. Published February 23, 2017. This paper is part of the JES Focus Issue on Mathematical Modeling of Electrochemical Systems at Multiple Scales in Honor of John Newman.

Fuel cell impedance can, in principle, give parameters of virtually all kinetic and transport processes running in the cell. Perhaps, there is no other experimental technique giving comparable to impedance amount of information on the cell function. However, understanding impedance spectra requires quite sophisticated modeling. Over the past decade, interest in impedance studies of fuel cells has been growing exponentially. Of particular interest are physical impedance models, ${ }^{1-18}$ as these models help to extract the transport and kinetic parameters from the cell impedance.

A major contribution to PEMFC impedance gives the cathode side. At small cell currents, the contribution of the oxygen transport in the gas-diffusion layer to the cell impedance is small. In PEMFCs at open circuit, this contribution is much less than the cathode catalyst layer (CCL) impedance due to hydrogen $\operatorname{crossover}^{19}$ (see also below). Thus, at small currents and in particular at open circuit voltage (OCV), the cell impedance spectrum depends on the processes in the CCL only.

Of special interest is the high-frequency (HF) impedance, as in this frequency domain, slow oxygen transport and faradaic processes are "frozen", i.e., they do not respond to the rapid variation of AC potential. The standard macrohomogeneous model shows that in the $\mathrm{HF}$ range, the impedance of a uniform CCL exhibits a straight line with the $45^{\circ}$ slope. ${ }^{2}$ This line is a signature of proton transport in the layer: projection of this line onto the real axis yields the CCL proton resistivity $R_{p}=l_{t} /\left(3 \sigma_{p}\right)$, which allows one to calculate the CCL proton conductivity $\sigma_{p}$ directly from the impedance spectrum. ${ }^{20,21}$ Here $l_{t}$ is the CCL thickness.

However, in a number of works, measured HF impedance strongly differs from the $45^{\circ}$ straight line. The HF part of the spectrum either has a form of an arc, which is independent of the cell current density, ${ }^{22,23}$ or it exhibits almost a straight line with the slope significantly exceeding $\pi / 4 .{ }^{24}$ Recently, Gerteisen ${ }^{17}$ developed a numerical model, which explains formation of these HF features by nonuniform CCL properties. In particular, the quasi-straight line has been explained in ${ }^{17}$ by exponentially decaying toward the GDL proton conductivity of the layer, while the current-independent arc forms as a concerted action of a nonuniform $\sigma_{p}$ and double layer capacitance $C_{d l}$. Malko et. al. ${ }^{24}$ explained the measured HF quasi-straight line in the spectrum of their electrode using a similar concept of impedance of a single pore with nonuniform Nafion coverage along the pore length.

An impedance study of PEMFC cathodes with possibly nonuniform Nafion loading has been reported by Lefebvre ${ }^{25}$ and $\mathrm{Li}$ and
Pickup. ${ }^{26}$ Both groups used an equivalent transmission line to fit the experimental spectra from several cathodes with different Nafion content. A rapidly decaying toward the GDL shape of the proton conductivity has been reported; much lower proton conductivity at the CCL/GDL interface was explained in $^{26}$ by penetration of catalyst to the pores of the GDL in combination with formation of isolated Nafion "islands" in this region. It should be noted that the experiments in Ref. 26 were performed under fixed cell potential and varying current density, depending on Nafion content.

Steady-state models ${ }^{27-29}$ and experiments $\mathrm{s}^{30-33}$ show that the CCL with growing toward the membrane Nafion content (proton conductivity) improves the cell performance. This explains interest in gradient electrodes from the fuel cell industry. Below, we develop analytical model of HF impedance of the CCL with nonuniform Nafion loading. We show that at high frequencies of the applied signal, the equations of transient macrohomogeneous model can be greatly simplified and analytical solution for the HF impedance can be obtained. Further, we use this solution for fitting impedance spectra of a PEMFC measured at open circuit conditions. We assume that the proton conductivity $\sigma_{p}$ decays exponentially through the CCL depth; the reference case of unform Nafion loading is described by the exponent with zero power. Fitting the model CCL impedance to the high-frequency points of the spectra gives the characteristic scale of $\sigma_{p}$ decay, the CCL proton conductivity at the membrane surface, and the double layer capacitance of the electrode. All these parameters are "reference" values at the open-circuit conditions.

\section{Model}

Basic equations.-Let the $x$-coordinate with the origin at the membrane surface be directed through the CCL depth (Figure 1). In this work, we assume that the proton conductivity is a function of $x$ :

$$
\sigma_{p}(x)=\sigma_{0} s(x)
$$

where $\sigma_{0}$ is the conductivity at the membrane interface (at $x=0$ ), and $s(\tilde{x})$ is the dimensionless shape function of $x$. The analysis below is based on the transient macrohomogeneous CCL model

$$
C_{d l} \frac{\partial \eta}{\partial t}+\frac{\partial j}{\partial x}=-2 i_{*}\left(\frac{c}{c_{r e f}}\right) \sinh \left(\frac{\eta}{b}\right)
$$

$$
j=-\sigma_{0} s(x) \frac{\partial \eta}{\partial x}
$$




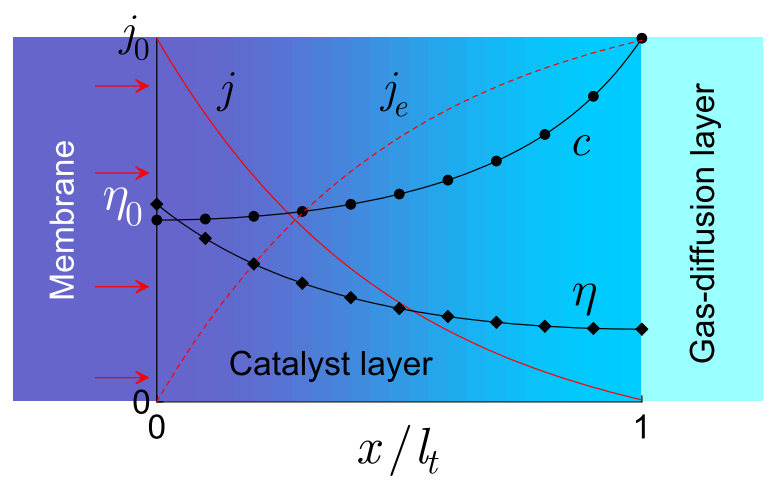

Figure 1. Schematic of the cathode catalyst layer and the $x$-coordinate. Note that the ORR overpotential $\eta$ is positive by convention. The gradient of Nafion loading is depicted as the gradient of blue color.

$$
\frac{\partial c}{\partial t}-D_{o x} \frac{\partial^{2} c}{\partial x^{2}}=-\frac{2 i_{*}}{4 F}\left(\frac{c}{c_{r e f}}\right) \sinh \left(\frac{\eta}{b}\right)
$$

Here, $C_{d l}$ is the double layer volumetric capacitance $\left(\mathrm{F} \mathrm{cm}^{-3}\right), \eta$ is the ORR overpotential, positive by convention, $t$ is time, $j$ is the local proton current density, $i_{*}$ is the volumetric exchange current density $\left(\mathrm{A} \mathrm{cm} \mathrm{cm}^{-3}\right), c$ is the local oxygen concentration, $c_{r e f}$ is its reference concentration, $b$ is the Tafel slope, $D_{o x}$ is the oxygen effective diffusion coefficient in the CCL. Eq. 2 is the proton charge conservation equation, Eq. 3 is the Ohm's law relating the proton current density to the gradient of overpotential, and Eq. 4 is the oxygen transport equation in the CCL with the Fick's law of diffusion. In the context of fuel cell electrode, the model above has been introduced and studied by Newman and Tobias. ${ }^{34}$ Detailed discussion of Eqs. $2-4$ is given in Ref. 35.

Substitution of Eq. 3 into Eq. 2 gives the diffusion-type equation for the overpotential. Linearization and Fourier-transform of the resulting system leads to the following pair of linear equations for the small-amplitude perturbations of overpotential $\eta^{1}$ and oxygen concentration $c^{1}$ (see ${ }^{35}$ for details):

$$
\begin{gathered}
\varepsilon^{2} \frac{\partial}{\partial \tilde{x}}\left(s(\tilde{x}) \frac{\partial \tilde{\eta}^{1}}{\partial \tilde{x}}\right)=\sinh \left(\tilde{\eta}^{0}\right) \tilde{c}^{1}+\left(\tilde{c}^{0} \cosh \tilde{\eta}^{0}+\mathrm{i} \tilde{\omega}\right) \tilde{\eta}^{1} \\
\varepsilon^{2} \tilde{D}_{o x} \frac{\partial^{2} \tilde{c}^{1}}{\partial \tilde{x}^{2}}=\left(\sinh \tilde{\eta}^{0}+\mathrm{i} \tilde{\omega} \mu^{2}\right) \tilde{c}^{1}+\tilde{c}^{0} \cosh \left(\tilde{\eta}^{0}\right) \tilde{\eta}^{1}
\end{gathered}
$$

where the superscripts 0 and 1 mark the steady-state solution and the amplitude of a small harmonic perturbation, respectively,

$$
\varepsilon=\sqrt{\frac{\sigma_{0} b}{2 i_{*} l_{t}^{2}}}, \quad \mu=\sqrt{\frac{4 F c_{r e f}}{C_{d l} b}}
$$

and the following dimensionless variables are used

$$
\begin{aligned}
\tilde{x} & =\frac{x}{l_{t}}, \quad \tilde{t}=\frac{t}{t_{*}}, \quad \tilde{\eta}=\frac{\eta}{b}, \quad \tilde{j}=\frac{j}{j_{p}}, \quad \tilde{c}=\frac{c}{c_{r e f}} \\
\tilde{D}_{o x} & =\frac{D_{o x}}{D_{*}}, \quad \tilde{Z}=\frac{Z \sigma_{0}}{l_{t}}, \quad \tilde{\omega}=\omega t_{*}
\end{aligned}
$$

Here $\omega=2 \pi f$ is the angular frequency of the applied signal,

$$
t_{*}=\frac{C_{d l} b}{2 i_{*}}, \quad j_{p}=\frac{\sigma_{0} b}{l_{t}}, \quad D_{*}=\frac{\sigma_{0} b}{4 F c_{r e f}}
$$

are the scaling parameters for time, current density, and diffusion coefficient, respectively.

The CCL impedance $\tilde{Z}$ is given by

$$
\tilde{Z}=-\left.\frac{\tilde{\eta}^{1}}{s(\tilde{x}) \partial \tilde{\eta}^{1} / \partial \tilde{x}}\right|_{\tilde{x}=0}
$$

High-frequency limit.- In this work we will focus on the highfrequency limit of Eqs. 5,6. In this limit, all the terms on the right side of Eq. 5, except $i \tilde{\omega} \tilde{\eta}^{1}$ can be neglected, and this equation simplifies to

$$
\varepsilon^{2} \frac{\partial}{\partial \tilde{x}}\left(s(\tilde{x}) \frac{\partial \tilde{\eta}^{1}}{\partial \tilde{x}}\right)=\mathrm{i} \tilde{\omega} \tilde{\eta}^{1}
$$

As can be seen, Eq. 11 does not contain $\tilde{c}^{1}$, which simply means that the oxygen concentration remains unperturbed if the frequency of the applied signal is high enough (the exact condition is formulated below). Eq. 11 thus decouples from the system. Note that Eq. 11 does not contain the static overpotential $\tilde{\eta}^{0}$, which means that this equation is valid for all cell currents, provided that the frequency of the applied signal is high.

Dividing both parts of Eq. 11 by $\varepsilon^{2}$ we get an equation

$$
\frac{\partial}{\partial \tilde{x}}\left(s(\tilde{x}) \frac{\partial \tilde{\eta}^{1}}{\partial \tilde{x}}\right)=\mathrm{i} \tilde{\Omega} \tilde{\eta}^{1}, \quad \tilde{\eta}^{1}(1)=\tilde{\eta}_{1}^{1},\left.\quad \frac{\partial \tilde{\eta}^{1}}{\partial \tilde{x}}\right|_{\tilde{x}=1}=0
$$

which depends on a single parameter, the reduced dimensionless frequency $\tilde{\Omega}$ :

$$
\tilde{\Omega}=\frac{\tilde{\omega}}{\varepsilon^{2}}=\frac{\omega C_{d l} l_{t}^{2}}{\sigma_{0}}
$$

Note that the factor $C_{d l} l_{t}^{2} / \sigma_{0}$ in Eq. 13 is the characteristic time for the relaxation of charge stored in the double layer. The first boundary condition in Eq. 12 fixes the amplitude of applied at $\tilde{x}=1$ perturbation, and the second condition means zero proton current through the CCL/GDL interface.

Eq. 12 can be transformed to a first-order equation for the local admittance $\tilde{Y}$. Introducing $\tilde{Y}(\tilde{x})$ according to

$$
\tilde{Y}=-\frac{s}{\tilde{\eta}} \frac{\partial \tilde{\eta}}{\partial \tilde{x}},
$$

substituting $-s \partial \tilde{\eta} / \partial \tilde{x}=\tilde{\eta} \tilde{Y}$ on the left side of Eq. 12, after simple transformations we get

$$
\frac{\partial \tilde{Y}}{\partial \tilde{x}}-\frac{\tilde{Y}^{2}}{s}=-\mathrm{i} \tilde{\Omega}, \quad \tilde{Y}(1)=0
$$

Here, the boundary condition follows from the second boundary condition in Eq. 12. From the definition of $\tilde{Y}$, Eq. 14, it follows that

$$
\tilde{Z}=\frac{1}{\tilde{Y}(0)}
$$

Useful solutions to Eq. 15 can be obtained if we guess some shape function $s(\tilde{x})$. Our experimental data (see below) suggest exponentially decaying toward the GDL $s(\tilde{x})$ :

$$
s(\tilde{x})=\exp (-\beta \tilde{x})
$$

where $\beta \geq 0$ is the inverse characteristic decay length. Note that $\beta=0$ describes the case of uniform Nafion loading. Solving Eq. 15 and calculating impedance, Eq. 16, we find

$$
\tilde{Z}_{H F}=\left(\sqrt{\frac{\mathrm{i}}{\tilde{\Omega}}}\right) \frac{Y_{0}\left(q \mathrm{e}^{\frac{\beta}{2}}\right) J_{1}(q)-Y_{1}(q) J_{0}\left(q \mathrm{e}^{\frac{\beta}{2}}\right)}{Y_{0}(q) J_{0}\left(q \mathrm{e}^{\frac{\beta}{2}}\right)-Y_{0}\left(q \mathrm{e}^{\frac{\beta}{2}}\right) J_{0}(q)}
$$

where

$$
q=\frac{2 \sqrt{-\mathrm{i} \tilde{\Omega}}}{\beta}
$$

and $J, Y$ are the Bessel functions of the first and second kind, respectively. In dimension form, this impedance reads

$$
Z_{H F}=\left(\sqrt{\frac{\mathrm{i}}{\omega \sigma_{0} C_{d l}}}\right) \frac{Y_{0}\left(q \mathrm{e}^{\frac{\beta}{2}}\right) J_{1}(q)-Y_{1}(q) J_{0}\left(q \mathrm{e}^{\frac{\beta}{2}}\right)}{Y_{0}(q) J_{0}\left(q \mathrm{e}^{\frac{\beta}{2}}\right)-Y_{0}\left(q \mathrm{e}^{\frac{\beta}{2}}\right) J_{0}(q)}
$$



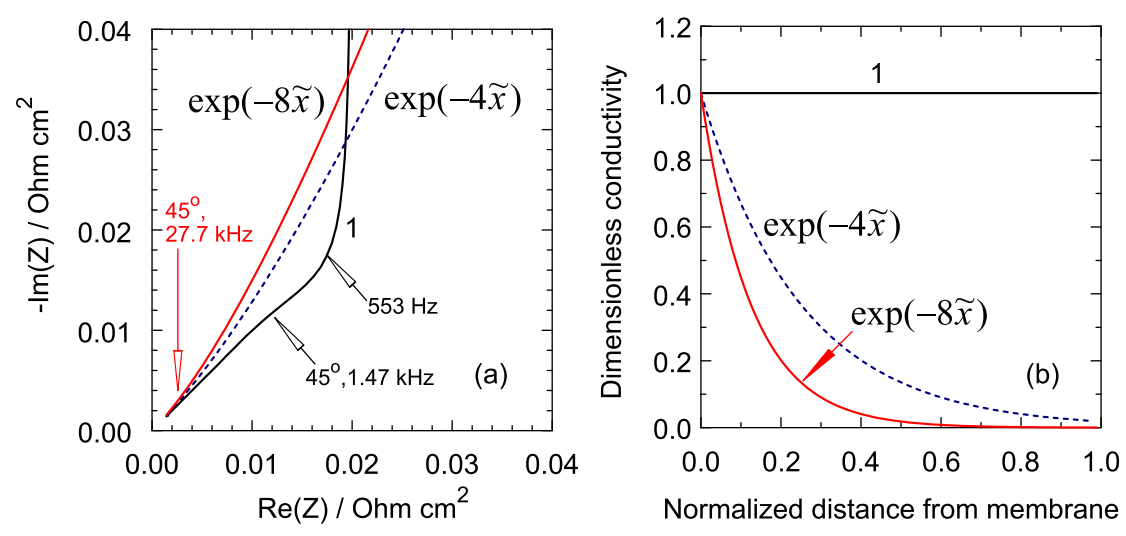

Normalized distance from membrane
Figure 2. (a) High-frequency part of model impedance spectra, Eq. 18, corresponding to uniform ionomer loading (1), and to exponentially decaying through the CCL depth proton conductivity ( $\beta=4$ and 8 ). (b) The respective shapes of the proton conductivity through the CCL depth. The other parameters for calculations are $\sigma_{0}=0.02 \Omega^{-1}$ $\mathrm{cm}^{-1}, C_{d l}=20 \mathrm{~F} \mathrm{~cm}^{-3}$, and $l_{t}=10^{-3} \mathrm{~cm}$. with

$$
q=\frac{2}{\beta} \sqrt{-\frac{i \omega C_{d l} l_{t}^{2}}{\sigma_{0}}},
$$

The limits of validity of Eq. 18 follow from a simple analysis of terms on the right side of Eq. 5. Clearly, $\tilde{c}^{1} \ll 1$ and close to the OCV, $\tilde{c}^{0} \simeq 1$; thus, the frequency-independent terms can be neglected if

$$
\tilde{\omega} \gg \cosh \tilde{\eta}^{0}
$$

or, in dimension form,

$$
\omega \gg \frac{2 i_{*} \cosh \left(\eta^{0} / b\right)}{C_{d l} b}
$$

At zero current in the load, the CCL still converts an equivalent current density of hydrogen crossover. In other words, in PEM fuel cells, true OCV conditions cannot be achieved and the cathode works in the Tafel regime even if the current in the load is zero. ${ }^{19}$ Thus, we may neglect the reverse exponent in Eq. 23, which leads to

$$
\omega \gg \frac{i_{*} \exp \left(\eta^{0} / b\right)}{C_{d l} b}
$$

As the oxygen concentration is close to the reference value, the Tafel equation reduces to $j_{\text {cross }}=l_{t} i_{*} \exp \left(\eta^{0} / b\right)$, where $j_{\text {cross }}$ is the equivalent current density of hydrogen crossover. Using this in Eq. 24, we finally find

$$
\omega \gg \frac{j_{\text {cross }}}{C_{d l} b l_{t}}
$$

It is advisable to estimate the right side of Eq. 25. A typical current density of hydrogen crossover is about $0.003 \mathrm{~A} \mathrm{~cm}^{-2}$. With $C_{d l}=20$ $\mathrm{F} \mathrm{cm}^{-3}, b=0.03 \mathrm{~V}$ and $l_{t}=10^{-3} \mathrm{~cm}$, we get $\omega \gg 5 \mathrm{~s}^{-1}$, which is equivalent to the regular frequencies

$$
f \gg 1 \mathrm{~Hz}
$$

Thus, Eq. 18 can be fitted to the part of the OCV spectrum above 10 $\mathrm{Hz}$. Note that though the validity condition Eq. 25 for the impedance (18) depends on $j_{\text {cross }}$, the impedance $\tilde{Z}_{H F}$ itself does not contain $j_{\text {cross }}$.

\section{Experimental}

The experiments have been performed using a segmented cell system developed at Hawaii Natural Energy Institute (HNEI) and GRandalytics test station. The segmented cell system employs close loop Hall sensors (Honeywell CSNN 191) for current sensing and allows us to perform simultaneous measurements of spatial electrochemical impedance spectroscopy (EIS), linear sweep voltammetry (LSV) and cyclic voltammetry (CV). The system details are provided in our previous papers. ${ }^{16,36}$

The segmented flow field of a cell consists of ten segments forming a continuous path along ten parallel serpentine channels. Each segment is equipped with its own current collector and GDL and it has an area of $7.6 \mathrm{~cm}^{2}$. The segmented hardware is applicable to either the anode or the cathode. The same channel designs are used for the segmented and the standard flow field on the anode side. The reactant streams were arranged in a co-flow configuration, and segment 1 is the inlet segment, and segment 10 is the outlet.

The cell was operated with commercially available $100 \mathrm{~cm}^{2}$ catalyst coated membrane from Gore with the thickness of $40-42 \mu \mathrm{m}$. The thickness of the reinforced membrane was $16-18 \mu \mathrm{m}$. The Pt/C loading of the anode and cathode electrodes was $0.4 \mathrm{mgPt} \mathrm{cm}{ }^{-2}$. Sigracet $25 \mathrm{BC}$ (thickness $235 \mu \mathrm{m}, 80 \%$ porosity) was used as the anode and cathode gas diffusion layers (GDLs). 25 BC consists of carbon paper substrate and microporous layer with the thickness of 40-45 $\mu \mathrm{m}$. Segmented GDL was used on the cathode side, whereas a uniform GDL was applied at the anode. The total active area of membrane/electrode assembly (MEA) was $76 \mathrm{~cm}^{2}$. The gasket material was made of Teflon, with the thicknesses of $125 \mu \mathrm{m}$ for the anode and cathode.

To perform EIS at open circuit voltage a 4-quandrant amplifier (4QA) has been employed instead of the normally used load bank. The 4QA can source and sink current, therefore it ensures that the average current over one perturbation period is truly zero. The segmented cell was assembled, conditioned and tested. The anode/cathode testing conditions for the EIS measurements were hydrogen/air at 1.0/1.0 1 $\min ^{-1}, 100 / 50 \%$ relative humidity and back pressure of $150 \mathrm{kPa}$. The cell temperature was $60^{\circ} \mathrm{C}$. The frequency range for the EIS was 0.1 $\mathrm{Hz}$ to $10 \mathrm{kHz}$ and the amplitude of the sinusoidal current perturbation corresponded to the amplitude of the cell voltage response of $10 \mathrm{mV}$ or lower. Spatial EIS are measured simultaneously from 10 segments and from the whole cell, thereby providing good statistics for fitting parameters (see below).

Hydrogen crossover current was measured by LSV using a Solartron SI 1287/electrochemical interface as a voltage source. The LSV was performed at the same operating conditions as EIS, while hydrogen and nitrogen were supplied to the reference/counter and working electrodes, respectively. The voltage sweep was applied from 0.1 to $0.4 \mathrm{~V}$ vs the reference hydrogen electrode at a scan rate of $0.1 \mathrm{mV} \mathrm{s}^{-1}$.

\section{Results and Discussion}

Figure 2 shows the high-frequency part of the model spectra, Eq. 18, corresponding to $\beta=0$ (uniform ionomer loading) and to exponentially decaying shapes of the proton conductivity $(\beta=4$ and $\beta=8$ ). As can be seen, with the frequency growth, the spectrum for $\beta=0$ forms a well-known straight line with the $45^{\circ}$ slope; however, the spectra corresponding to $\beta=4$ and 8 look like a straight line with larger slope, which increases with $\beta$. A more detailed view of this effect is depicted in Figure 3, which shows that the phase angle of impedance Eq. 18 increases with $\beta$, though it tends to $45^{\circ}$ as $\omega \rightarrow \infty$. Note that the phase angle of the uniformly-loaded spectrum changes rapidly in the frequency range $\omega \simeq 10^{2}-10^{4} \mathrm{~s}^{-1}$, and it reaches the 


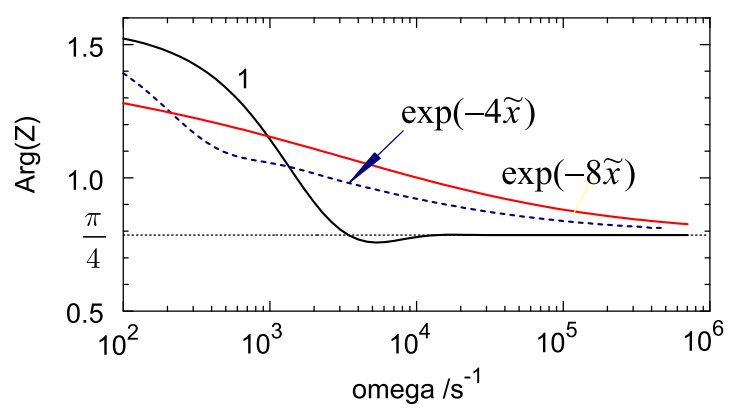

Figure 3. Bode plot of the phase angle of the model high-frequency impedance for the three indicated shapes of the proton conductivity through the CCL thickness $x$. The larger the gradient of $\sigma_{p}$ along $x$, the larger the phase angle of the HF part of the spectrum. Note that the phase angles for $\beta=4$ and 8 vary rather slowly with $\omega$, which gives an impression that the respective part of the Nyquist spectrum is close to the straight line (see Figure 2a).

value of $45^{\circ}$ at $\omega \simeq 10^{4} \mathrm{~s}^{-1}$. In contrast, the phase angle of the other two spectra in Figure 3 vary rather slowly in the range of $\omega \simeq 10^{3}$ $10^{5} \mathrm{~s}^{-1}$, and they approach the $45^{\circ}$-slope asymptotically at $\omega>10^{6}$ $\mathrm{s}^{-1}$, which is typically out of the frequency range used in impedance studies of fuel cells (Figure 3).

Next, Eq. 18 has been fitted to the HF part of experimental spectra. The spectra have been measured at zero current in the external load; as discussed above, this corresponds to the CCL operation at the current density of hydrogen crossover. This current was measured to be about $3 \mathrm{~mA} \mathrm{~cm}^{-2}$, which limits validity of Eq. 18 by the frequency $f \simeq 10 \mathrm{~Hz}$.

The fitting has been performed in Maple environment using a built-in Maple procedure NonlinearFit. The experimental and fitted curves for the whole cell and for the segments 1 to 3 are shown in Figure 4. The parameters resulted from fitting are depicted in Figure 5; their mean over the cell surface values are summarized in Table I. As can be seen, the values of $\beta$ group quite well around the mean of 7.3 (Figure 5a). Large $\beta$ indicates quite strong nonuniformity of the proton conductivity through the CCL depth. The proton conductivity at the membrane interface $\sigma_{0}$ varies in the range from 0.09 to $0.23 \Omega^{-1}$ $\mathrm{cm}^{-1}$ (Figure $5 \mathrm{~b}$ ). This spread can be explained by "fuzzy" boundary between the CCL and membrane. However, the mean value of $\sigma_{0}$ of $0.13 \Omega^{-1} \mathrm{~cm}^{-1}$ agrees well with the conductivity of fully humidified Nafion at $60^{\circ} \mathrm{C} .{ }^{37}$ The mean over the cell surface shape of the proton conductivity through the CCL depth is depicted in Figure 6. It is worth noting that the average over $x$ proton conductivity is $0.018 \Omega^{-1}$ $\mathrm{cm}^{-1}$, which correlates with the literature data. ${ }^{20}$ The double layer capacitance is $28 \pm 2 \mathrm{~F} \mathrm{~cm}^{-3}$ (Figure $5 \mathrm{c}$ and Table I). This value is in good agreement with that obtained from impedance at a finite cell current density. ${ }^{38}$

A very few literature data exist on impedance of gradient electrodes (see introduction section). The reason is that making a reproducible electrode with the prescribed shape of Nafion loading is only possible using an expensive spray coating machines. These machines are

\footnotetext{
Table I. The cell parameters resulted from impedance fitting; the hydrogen crossover current density $j_{\text {cross }}$ was measured by linear sweep voltammetry and the CCL thickness $l_{t}$ was obtained by scanning electron microscopy.

Characteristic scale of the exponent in $7.3 \pm 0.4$

Eq. $17 \beta$

CCL proton conductivity at the membrane

interface $\sigma_{0}, \Omega^{-1} \mathrm{~cm}^{-1}$

Double layer capacitance $C_{d l}, \mathrm{~F} \mathrm{~cm}^{-3}$

Catalyst layer thickness $l_{t}, \mathrm{~cm}$

Current density of hydrogen crossover

$0.12 \pm 0.03$

$28 \pm 2$

$0.0012 \pm 0.0004$

$3.1 \pm 0.5$
}

$j_{\text {cross }}, \mathrm{mA} \mathrm{cm}^{-2}$
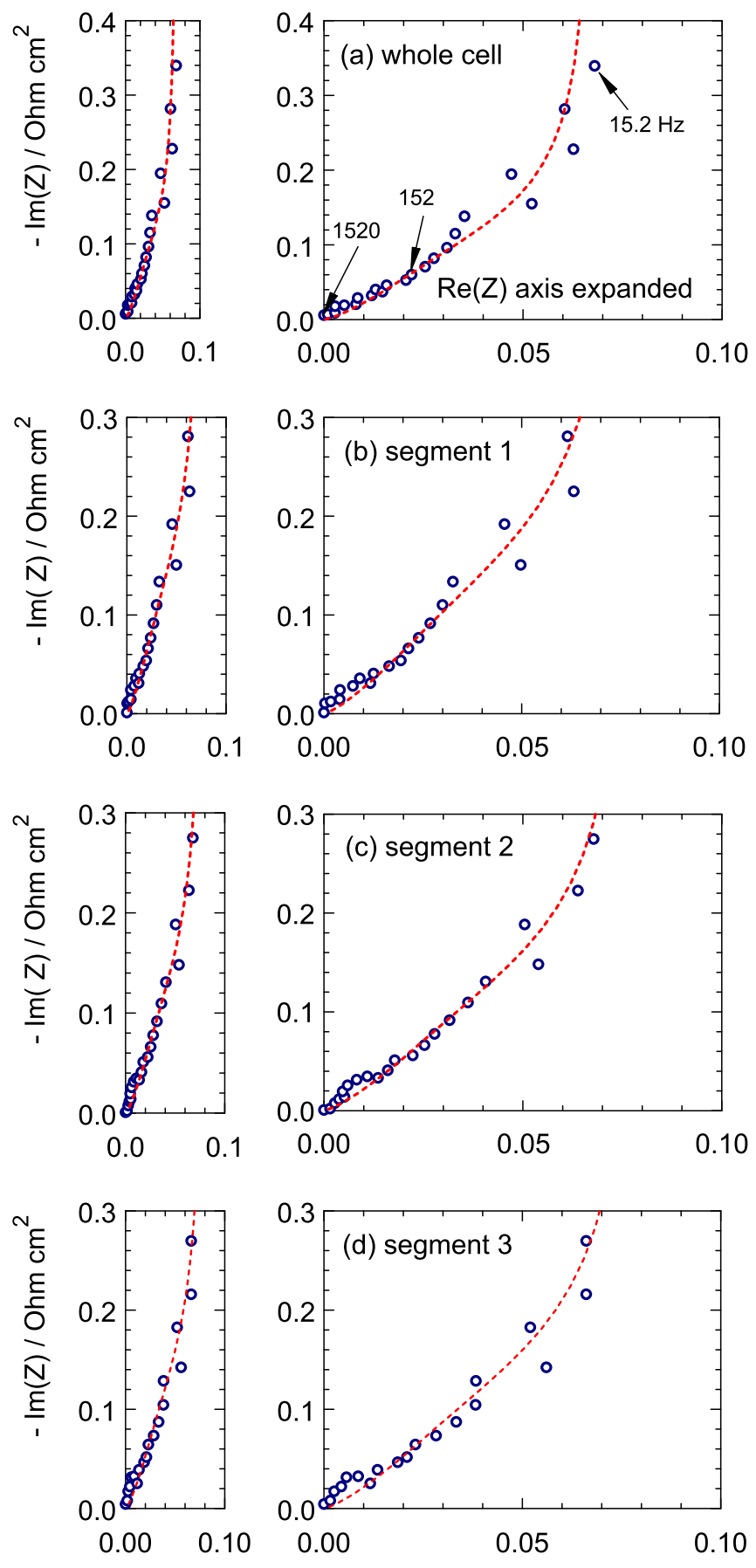

$\operatorname{Re}(\mathrm{Z}) / \mathrm{Ohm} \mathrm{cm}{ }^{2}$

$\operatorname{Re}(\mathrm{Z}) / \mathrm{Ohm} \mathrm{cm} \mathrm{cm}^{2}$

Figure 4. Experimental (points) and fitted model impedance (dashed lines) for the whole cell and for the segments 1 to 3 . The left panels show the Nyquist spectra in the coordinates with equal scales along the real and imaginary axis. The right panels show the same spectra with the stretched real coordinate, to represent the details. Arrows in the frame (a) indicate frequencies $f, \mathrm{~Hz}$.

available on the market, however, not many labs have this equipment. It seems that traditional methods of electrode preparation lead to uncontrollable gradients of a Nafion content during drying or thermal processing..$^{25,26}$

The advantage of the HF@OCV technique discussed in this work is twofold. First, in typical PEMFC, it can be used for analysis of impedance at the frequencies above $10 \mathrm{~Hz}$. The standard frequency range in fuel cell impedance studies extends up to $10 \mathrm{kHz}$; thus, 


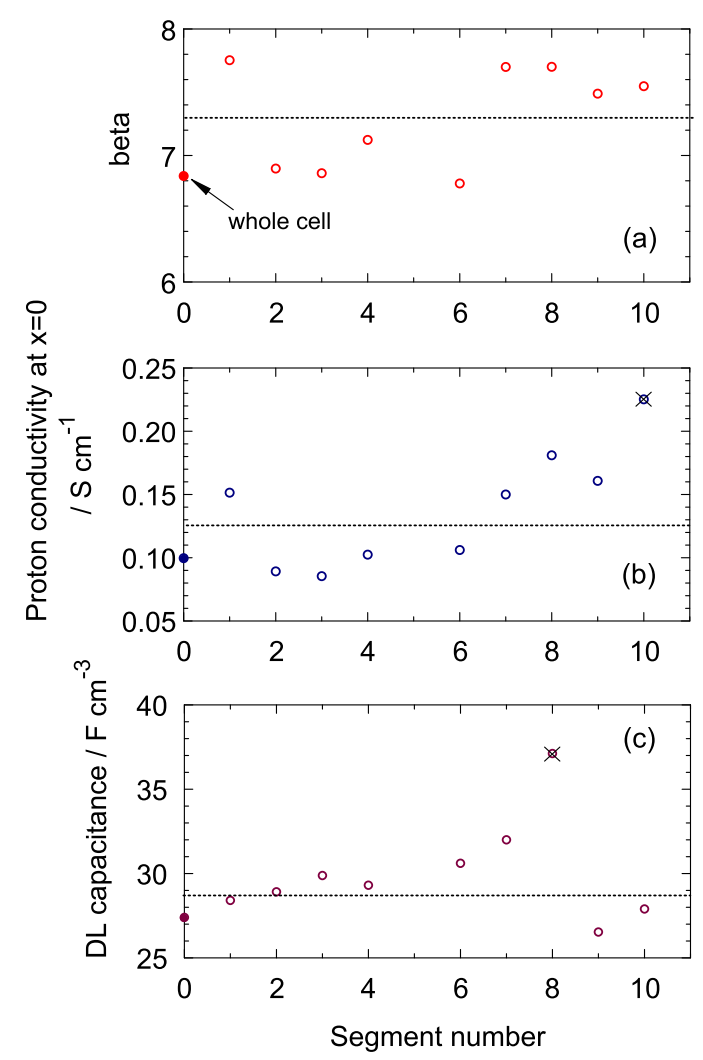

Figure 5. The fitting parameters for individual segments. Parameters for the whole cell are depicted as zero segment (filled circles). The parameters excluded from calculation of mean values are marked by crosses.

the method above is applicable to the OCV impedance data in the frequency window covering three decades, from $10 \mathrm{~Hz}$ to $10 \mathrm{kHz}$. This greatly improves reliability of the method. Second, in the HF domain, the inertial oxygen transport and faradaic processes are "frozen", i.e., the signal changes so fast, that these processes do not respond. This is the best situation to determine the transport parameter for ions, which respond immediately to the HF potential, and the double layer capacitance, which is being charged by the ions. Last but not least, the method provides "reference" values of $\beta, \sigma_{0}$ and $C_{d l}$ at zero cell current density, when the amount of water in the CCL is determined solely by external humidification of the cathode stream.

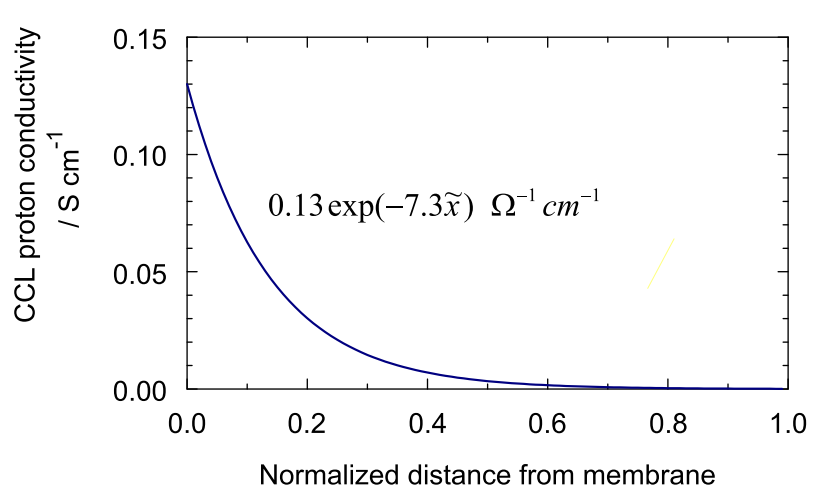

Figure 6. The averaged over the cell surface shape of the CCL proton conductivity through the CCL depth.

\section{Conclusions}

A model for the high-frequency impedance of the cathode catalyst layer with nonuniform Nafion loading is developed. Under the assumption of exponential decay of the CCL proton conductivity $\sigma_{p}$ from the membrane surface, an analytical solution for the CCL impedance is derived. The impedance exhibits a quasi-straight line in the HF range, with the slope of the line depending on the characteristic scale of $\sigma_{p}$ decay along the $x$-coordinate through the CCL depth. The model impedance is fitted to the HF part of the experimental Nyquist spectra obtained from the segmented PEM fuel cell at the open-circuit conditions. Fitting gives the characteristic scale of $\sigma_{p}$ decay along $x$, the CCL proton conductivity at the membrane interface, and the double layer capacitance $C_{d l}$.

\section{Acknowledgments}

T. Reshetenko is grateful to funding from US Office of Naval Research (N00014-11-1-0391), US Army Research Office (W911NF15-1-0188) and the Hawaiian Electric Company for ongoing support of the Hawaii Sustainable Energy Research Facility. The authors also thank Gunter Randolf for valuable support regarding the system operation. The authors are grateful to Professor John Newman for his outstanding contributions to the theory of fuel cells, and in particular, to the theory of PEM fuel cell impedance.

\section{Lists of Symbols} $b$
Marks dimensionless variables

ORR Tafel slope, $\mathrm{V}$

Double layer volumetric capacitance, $\mathrm{F} \mathrm{cm}^{-3}$

Oxygen molar concentration in the CCL, $\mathrm{mol} \mathrm{cm}^{-3}$

Reference oxygen molar concentration, $\mathrm{mol} \mathrm{cm} \mathrm{cm}^{-3}$

Effective oxygen diffusion coefficient in the CCL, $\mathrm{cm}^{2} \mathrm{~s}^{-1}$

Faraday constant, $\mathrm{C} \mathrm{mol}^{-1}$

Regular frequency, $\mathrm{Hz}$

Local cell current density, $\mathrm{A} \mathrm{cm}^{-2}$

Current density of hydrogen crossover, $\mathrm{A} \mathrm{cm}^{-2}$

Imaginary unit

Volumetric exchange current density, $\mathrm{A} \mathrm{cm}^{-3}$

Catalyst layer thickness, cm

Auxiliary parameter, Eq. 19

Dimensionless shape of the CCL proton conductivity, Eq. 17

Proton resistivity of the CCL, $\Omega \mathrm{cm}^{2}$

Time, $\mathrm{s}$

Characteristic time of double layer charging, s, Eq. 9

Coordinate through the CCL, cm

CCL impedance, $\Omega \mathrm{cm}^{2}$

High-frequency CCL impedance, $\Omega \mathrm{cm}^{2}$

\author{
Subscripts \\ Membrane/CCL interface \\ CCL/GDL interface \\ High-frequency \\ Catalyst layer \\ Characteristic value
}

\section{Superscripts}

Steady-state value

Small-amplitude perturbation

\section{Greek}

Characteristic parameter of $\sigma_{p}$ decay, Eq. 17

Newman's dimensionless reaction penetration depth, Eq. 7 
$\mu \quad$ Dimensionless parameter, Eq. 7

$\sigma_{p} \quad$ CCL ionic conductivity, $\Omega^{-1} \mathrm{~cm}^{-1}$

$\sigma_{0}$ CCL ionic conductivity at the membrane surface, $\Omega^{-1} \mathrm{~cm}^{-1}$

$\tilde{\Omega} \quad$ Reduced dimensionless frequency, Eq. 13

$\omega$

\section{References}

1. T. E. Springer, T. A. Zawodzinski, M. S. Wilson, and S. Gottesfeld, Characterization of polymer electrolyte fuel cells using AC impedance spectroscopy. J. Electrochem. Soc., 143, 587, (1996)

2. M. Eikerling and A. A. Kornyshev, Electrochemical impedance of the cathode catalyst layer in polymer electrolyte fuel cells. J. Electroanal. Chem., 475, 107, (1999).

3. Y. Bultel, L. Genies, O. Antoine, P. Ozil, and R. Durand, Modeling impedance diagrams of active layers in gas diffusion electrodes: Diffusion, ohmic drop effects and multistep reactions. J. Electroanal. Chem., 527, 143 (2002).

4. F. Jaouen and G. Lindbergh, Transient techniques for investigating mass-transport limitations in gas diffusion electrode. J. Electrochem. Soc., 150, A1699 (2003).

5. Q. Guo and R. E. White, A steady-state impedance model for a PEMFC cahode. $J$. Electrochem. Soc., 151, E133 (2004).

6. Y. Bultel, K. Wiezell, F. Jaouen, P. Ozil, and G. Lindbergh, Investigation of mass transport in gas diffusion layer at the air cathode of a PEMFC. Electrochimica Acta, 51, 474 (2005).

7. D. Gerteisen, A. Hakenjos, and J. O. Schumacher, AC impedance modeling study on porous electrodes of proton exchange membrane fuel cells using an agglomerate model. J. Power Sources, 173, 346 (2007).

8. A. A. Franco, P. Schott, C. Jallut, and B. Maschke, A multi-scale dynamic mechanistic model for the transient analysis of PEFCs. Fuel Cells, 7, 99 (2007).

9. M. Cimenti, D. Bessarabov, M. Tam, and J. Stumper, Investigation of proton transport in the catalyst layer of PEM fuel cells by electrochemical impedance spectroscopy. ECS Transactions, 28(23), 147 (2010).

10. I. A. Schneider, M. H. Bayer, and S. von Dahlen, Locally resolved electrochemical impedance spectroscopy in channel and land areas of a differential polymer electrolyte fuel cell. J. Electrochem. Soc., 158, B343 (2011).

11. J. Mainka, G. Maranzana, A. Thomas, J. Dillet, S. Didierjean, and O. Lottin, Onedimensional model of oxygen transport impedance accounting for convection perpendicular to the electrode. Fuel Cells, 12, 848 (2012)

12. J. R. Vang, S. J. Andreasen, and S. K. Kaer, A transient fuel cell model to simulate HTPEM fuel cell impedance spectra. J. Fuel Cell Sci. Techn., 9, 021005, (2012).

13. A. A. Kulikovsky, Analytical solutions for polarization curve and impedance of the cathode catalyst layer with fast oxygen transport in a PEM fuel cell. J. Electrochem. Soc., 161, E3171 (2014).

14. A. A. Kulikovsky, Exact low-current analytical solution for impedance of the cathode catalyst layer in a PEM fuel cell. Electrochimica Acta, 147, 773 (2014).

15. A. A. Kulikovsky, One-dimensional impedance of the cathode side of a pem fuel cell: Exact analytical solution. J. Electrochem. Soc., 162, F217 (2015).

16. T. Reshetenko and A. Kulikovsky, PEM fuel cell characterization by means of the physical model for impedance spectra. J. Electrochem. Soc., 162, F627 (2015).

17. D. Gerteisen, Impact of inhomogeneous catalyst layer properties on impedance spectra of polymer electrolyte membrane fuel cells. J. Electrochem. Soc., 162, F1431 (2015).

18. A. A. Kulikovsky, A simple physics-based equation for low-current impedance of a PEM fuel cell cathode. Electrochimica Acta, 196, 231 (2016).

19. A. A. Kulikovsky, PEM fuel cell impedance at open circuit. J. Electrochem. Soc., 163, F319 (2016)

20. , R Makharia, M. F. Mathias, and D. R. Baker, Measurement of catalyst layer electrolyte resistance in PEFCs using electrochemical impedance spectroscopy. J. Electrochem. Soc., 152, A970 (2005).

21. A. A. Kulikovsky and M. Eikerling, Analytical solutions for impedance of the cathode catalyst layer in PEM fuel cell: Layer parameters from impedance spectrum without fitting. J. Electroanal. Chem., 691, 13 (2013).

22. M Keddam, C Rakotomavo, and H. Takenouti, AC impedance diagnosis of a 500W PEM fuel cell stack Part I: Stack impedance. J. Power Sources, 161, 920 (2006)

23. D Malevich, E Halliop, B. A. Peppley, J. G. Pharoah, and K. Karan, Investigation of charge-transfer and mass-transport resistances in pemfcs with microporous layer using electrochemical impedance spectroscopy. J. Electrochem. Soc., 156, B216 (2009).

24. D Malko, T Lopes, E. A. Ticianelli, and A. Kucernak, A catalyst layer optimisation approach using electrochemical impedance spectroscopy for PEM fuel cells operated with pyrolysed transition metal-N-C catalysts. J. Power Sources, 323, 189 (2016).

25. M. C. Lefebvre, R. B. Martin, and P. G. Pickup, Characterization of ionic conductivity profiles within proton exchange membrane fuel cell gas diffusion electrodes by impedance spectroscopy. Electrochem. Solid State Lett., 2, 259 (1999).

26. G. Li and P. G. Pickup, Ionic conductivity of pemfc electrodes. effect of Nafion loading. J. Electrochem. Soc., 150, C745 (2003).

27. Q Wang, M Eikerling, D Song, Zh. Liu, T Navessin, Zh. Xie, and S. Holdcroft, Functionally graded cathode catalyst layers for polymer electrolyte fuel cell. J. Electrochem. Soc., 151, A950 (2004).

28. P. P. Mukherjee and C.-Y. Wang, Direct numerical simulation modeling of bilayer cathode catalyst layers in polymer electrolyte fuel cells. J. Electrochem. Soc., 154, B1121 (2007).

29. A. A. Kulikovsky, A model for optimal catalyst layer in a fuel cell. Electrochimica Acta, 79, 31 (2012).

30. Y.-G. Yoon, T.-H. Yang, G.-G. Park, W.-Y. Lee, and C.-S. Kim, A multi-layer structured cathode for the PEMFC. J. Power Sources, 118, 189 (2003).

31. D Song, Q Wang, Z Liu, M Eikerling, Z Xie, T Navessin, and S. Holdcroft, A method for optimizing distributions of Nafion and Pt in cathode catalyst layers of PEM fuel cells. Electrochimica Acta, 50, 3347 (2005).

32. K.-H. Kim, H.-J. Kim, K.-Y. Lee, J. H. Jang, S.-Y. Lee, E. Cho, I.-H. Oh, and T.-H. Lim, Effect of Nafions gradient in dual catalyst layer on proton exchange membrane fuel cell performance. Int. J. Hydrogen Energy, 33, 2783 (2008).

33. D. Lee and S. Hwang, Effect of loading and distributions of Nafion ionomer in the catalyst layer for PEMFCs. Int. J. Hydrogen Energy, 33, 2790 (2008).

34. J. S. Newman and C. W. Tobias, Theoretical analysis of current distribution in porous electrodes. J. Electrochem. Soc., 109, 1183 (1962).

35. A. A. Kulikovsky, A physical model for the catalyst layer impedance. J. Electroanal. Chem., 669, 28 (2012).

36. T. Reshetenko and A. Kulikovsky, Comparison of two physical models for fitting PEM fuel cell impedance spectra measured at a low air flow stoichiometry. J. Electrochem. Soc., 163, F238 (2016).

37. T. A. Zawodzinski, M Neeman, L. O. Sillerud, and S. Cottesfeld, Determination of water dlffusion coefficients in perfluorosulfonate ionomeric membranes. J. Phys. Chem., 95, 6040 (1991).

38. T. Reshetenko and A. Kulikovsky, Variation of PEM fuel cell physical parameter with current: Impedance spectroscopy study. J. Electrochem. Soc., 163(9), F1100 (2016) 